\title{
Gross calorific value estimation for milled maize cob biomass using near infrared spectroscopy
}

\author{
Jetsada Posom ${ }^{1,2}$ and Natrapee Nakawajana ${ }^{3, *}$ \\ ${ }^{1}$ Department of Agricultural Engineering, Faculty of Engineering, Khon Kaen University, Khon Kaen, 40002, Thailand. \\ ${ }^{2}$ Applied Engineering for Important Crops of the North East Research Group, Department of Agricultural Engineering, Faculty of \\ Engineering, Khon Kaen University 40002, Thailand. \\ ${ }^{3}$ Department of Agricultural Engineering, Faculty of Engineering, King Mongkut's Institute of Technology Ladkrabang. 1 \\ Chalongkrung Road, Ladkrabang, Bangkok 10520, Thailand.
}

\begin{abstract}
The maize cob biomass is one of important biomass crops in Thailand. Nowadays, the use of the biomass as renewable resource is increasing, especially residue agriculture waste. As we know that the biomass properties impact combustion, in order to achieve boiler efficiency, its energy characteristics of biomass was required immediately before burning. This work uses the FT-near infrared spectroscopy to estimate gross calorific value (GCV) of maize cob as the rapid method. Each sample was scanned using diffuse reflectance mode at a wavenumber range between $12500-3600 \mathrm{~cm}^{-1}$. The scanning was done with a resolution of $8 \mathrm{~cm}^{-1}$ and completed 32 scans per sample, then averaged to be one spectrum. The results showed that this technique could decrease a processing time to 1-2 minutes per sample to determine GCV whereas alternatively the current method used a processing time of 25-30 minutes per sample. The capacity of the model gave root mean square error of cross validation (RMSECV) of $91.1 \mathrm{Jg}^{-1}$, which was low. Hence, the model was acceptable and cloud be used for screening.
\end{abstract}

\section{Introduction}

Maize cob is a waste from agricultural process after the seed is removed from the cob. Maize cob can then become a habitat of bird and rat and moreover, bad smell could happen as well. This is the important problem of traders. Previously, the maize cob was used as a fertilizer but it was not popular for farmer because it takes a long time for decomposition. Hence, it has been initiated to use the maize cob as a fuel.

Thailand has imported maize cob. It's a waste of agricultural product from one of the economic crops of Thailand as a biomass fuel. When the maize cob is burned, it has calorific value approximately 17,000 $\mathrm{kJ} / \mathrm{kg}$, and it takes a long time to burn before becoming the ash. This implies that it has a characteristic of good fuel.

The major aspect of waste residue properties to be renewable energy is the gross calorific value (GCV) which is also known as the higher heating value. GCV is total energy in biomass released during burning process, which takes into account the latent heat of vaporization of water in the combustion products. Normally, GCV can be measured by bomb calorimeter but this method takes a processing time about 25-30 minutes per sample for measuring and is subject to high cost of analysis.

Near infrared (NIR) spectroscopy is a nondestructive and rapid technique for estimating a quality of food and agricultural products., In addition, there have been some reports that demonstrated the use of NIR spectroscopy for the evaluation of the GCV and other properties of biomass such as the evaluation of heating value of bamboo [1], the evaluation of moisture content and GCV of Leucaena leucocephala pellets [2], the prediction of GCV of Miscanthus [3], the GCV prediction of straw [4], the evaluation of moisture content and the GCV of oil-extracted residue of Jatropha curcas [9] and the analysis of GCV and elemental compositions of sorghum [10]. The results of their studies suggested that NIR spectroscopy had the potential to estimate the calorific value of biomass fuel. Hence, the objective of this research was to assess the potential of NIR spectroscopy to estimate GCV of milled maize cob biomass.

\section{Materials and Methods}

\subsection{Sample}

60 maize cob samples were collected from different area. The 60 samples were divided into two groups, 50 samples were used for utilizing calibration model and 10 samples were used as unknown sample for testing the calibration model. After harvest each sample from a plant, they were crushed into $5 \mathrm{~cm}$ and then dried to a constant weight by hot air oven (Memmert, model ULM 500 , Germany) at $105{ }^{\circ} \mathrm{C}$ for $24 \mathrm{~h}$, this process was

\footnotetext{
* Corresponding author: natrapee.na@kmitl.ac.th
} 
preparing sample for easy milling in next process. Then, all sample were milled by milling machine (FRITSCH, $14.3000 / 10857$, Germany) through a hole sizes of 2.00 $\mathrm{mm}$ and kept in an aluminium bag prior to the experiment. Next, each sample was measured the NIR absorbance using FT-NIR spectrometer and then some part of sample was measured the GCV by bomb calorimeter and other part was measured the moisture content for reference data by hot air oven at $105{ }^{\circ} \mathrm{C}, 24$ $\mathrm{h}$. The averaging moisture content of 60 samples were around $9.43 \%$ dry basis.

\subsection{NIR spectral acquisition}

Each milled maize cob sample was poured into a quartz bottom sample cup having dimension of $43 \mathrm{~mm}$ in diameter and $50 \mathrm{~mm}$ in height. For the reflectance mode, the thickness of sample was confirmed as infinity and must be ensured that there was no light leaked or transmitted through the sample. Fourier-transform infrared spectroscopy (FTNIR) (MPA, Bruker, Germany) was applied for scanning and absorbance was derived in $\log 1 / \mathrm{R}$ unit. Scanning conditions involved a resolution of $8 \mathrm{~cm}^{-1}$, wavenumber range of 12500-3600 $\mathrm{cm}^{-1}$, and the number of scanning of 32 .

\subsection{Reference data}

After the milled maize cob samples had been scanned, the milled maize cob was sampled from the bottom of cup due to direct absorption by NIR radiation. About 0.5 $\mathrm{g}$ of sample was pelletized and the GCV was determined using a bomb calorimeter (C200, IKA, Germany) in isoperibol mode. The bomb calorimeter was calibrated by pelletized benzoic acid (IKA C 723, IKA, Germany).

After the GCV had been measured, the outliers were then checked by $\left(\left(x_{i}-x \overline{)}\right) / S D \geq \pm 3\right.$, where $x_{i}$ is the reference data of sample $i . x$ and SD are the average and standard deviation of the reference data. If it was found, it was then rejected from the data set and was not used for modelling.

\subsection{Data processing and NIR spectroscopy modelling}

The NIR spectra and its corresponding reference data of GCV of fifty samples were used for modelling. The NIR models for predicting the GCV were constructed by PLS regression and validated by full cross validation. The spectral pre-treatment and modelling were carried on by OPUS software, version 7.0.129, Germany. Before the model development, the NIR spectra were pre-processed by constant offset elimination, straight line subtraction, vector normalization, min-max normalization, MSC (multiplicative scatter correction), first derivatives, second derivatives, the combination between first derivatives and straight line subtraction, the combination between first derivatives and vector normalization and the combination between first derivatives and MSC.

The effective model was obtained by lowest root mean squares error of cross validation (RMSECV), from which the number of PLS factor, spectral pre-treatment technique, and wavenumber range were listed. After that, the regression coefficient versus optimal wavenumber range was plotted. After modelling, the external sample of ten were used for tested the PLS model.

The performance of the GCV predicting model was stated by determination of the coefficient of determination $\left(\mathrm{R}^{2}\right)$, bias, root mean square error of cross validation (RMSECV) and ratio of standard error of cross-validation to deviation (RPD).

\section{Results and discussions}

\subsection{Ground maize cob spectra}

The raw spectra of 60 milled maize cob biomass samples in a range of $12500-3600 \mathrm{~cm}^{-1}$ were illustrated in Fig. 1. In the raw spectra of milled maize cob sample, the main absorption bands were observed at $5820(1718 \mathrm{~nm})$, $5180(1930 \mathrm{~nm}), 4408(2268 \mathrm{~nm})$, and $4266 \mathrm{~cm}-1$ (2344 $\mathrm{nm})$, corresponding to $\mathrm{C}-\mathrm{H}$ stretching first overtone of hydrocarbon [5], O-H stretching/ $\mathrm{HOH}$ deformation combination of starch [6], O-H stretching C-O stretch combination of cellulose [6], and $\mathrm{C}-\mathrm{H}$ methylene of hydrocarbon [5].

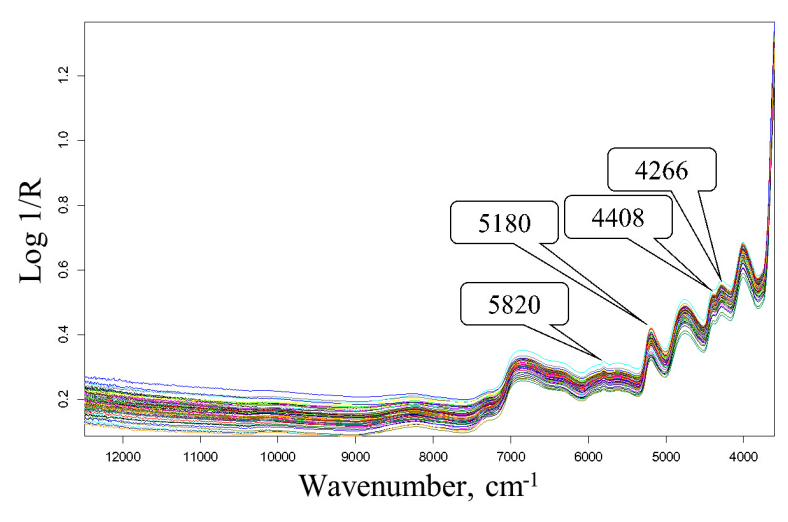

Fig. 1. The raw spectra of 60 milled maize cob biomass samples across the wavenumber of 12500 to $3600 \mathrm{~cm}^{-1}$

\subsection{Reference data of the GCV}

Statistical data of calibration set, and external sample set for GCV of rice husk was demonstrated in Table 1 which shows the maximum, minimum, average, and standard deviation (SD). The maximum and minimum value of calibration set was higher and lower than external sample set, respectively, which covering the external sample. Then the calibration set can be representative sample for future prediction.

Table 1. Statistical values of gross calorific value data of maize cob milled samples used in model development.

\begin{tabular}{|c|c|c|}
\hline & Calibration set & External sample \\
\hline $\operatorname{Max}) \mathrm{Jg}^{-1}($ & 17800.5 & 17431.0 \\
\hline $\operatorname{Min}\left(\mathrm{Jg}^{-1}\right)$ & 17005.0 & 17253.0 \\
\hline Mean $\left(\mathrm{Jg}^{-1}\right)$ & 17405.0 & 17351.2 \\
\hline $\mathrm{SD}\left(\mathrm{Jg}^{-1}\right)$ & 178.2 & 62.9 \\
\hline
\end{tabular}




\subsection{Near infrared spectroscopy models for GCV of milled maize cob sample}

The effective calibration model was optimized using the wavenumber range of 5450.2-4246.7 $\mathrm{cm}^{-1}$. The spectral pre-treatment of second derivative and the number of PLS factor listed from 1 to 3 was used. The PLS factor of optimal model was selected which gave the lowest RMSECV. Fig. 2a. displays the RMSEE versus PLS factor for calibration set whereas RMSECV versus PLS factor for validation set is shown in Fig. 2b. The PLS factor of three gave the lowest RMSECV which was then used for modelling.

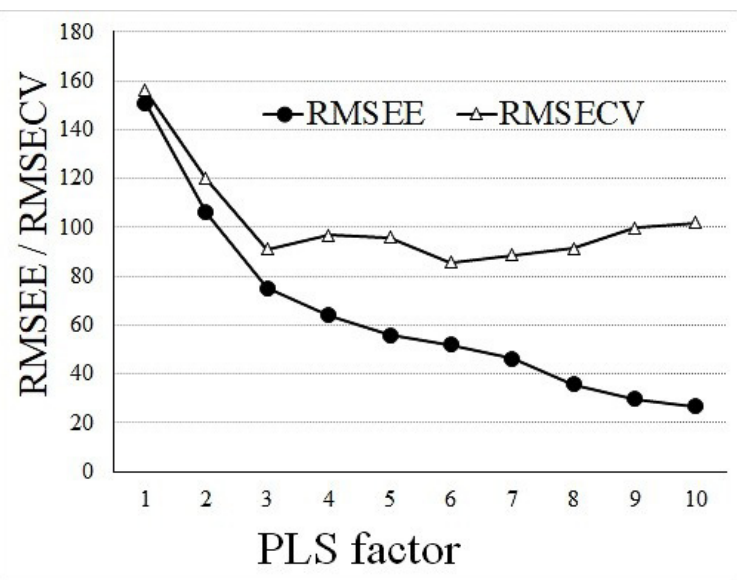

Fig. 2. RMSEE/RMSECV vs rank (PLS factor) for calibration set, and validation set

The PLS model was tested using leave-one-out cross-validation providing $\mathrm{R}^{2}$, RMSEE, $\mathrm{r}^{2}$, RMSECV, RPD, and bias of $0.83,75 \mathrm{Jg}^{-1}, 0.73,91 \mathrm{Jg}^{-1}, 1.94$, and $0.293 \mathrm{Jg}^{-1}$, respectively. Zornoza et al. [7] recommended that if $\mathrm{R}^{2}$ and RPD are between 0.66 to 0.80 and 2.0 to 2.5, respectively, it permitted only approximate prediction and could be used with screening and some other "approximate" calibrations [8]. The $\mathrm{R}^{2}$ and RPD provided were less than 0.66 and 2 , respectively, which meant the A content was poorly predicted [8]. William [8] suggested that $R^{2}$ between 0.50 and 0.64 could be used for rough screening. The ratio of bias to its mean of measured value were $0.0165 \%\left(0.293 \mathrm{Jg}^{-1} \div 17405.0 \mathrm{Jg}^{-1}\right)$ for GCV, which was very small. So that, we recommended that the models were suitable for estimation of the GCV of milled maize cob sample.

$\mathrm{R}^{2}$ stated the percentage of proportion of the variance in GCV that can be explained by variance in absorption value [26]. For example, if $\mathrm{R}^{2}$ was 0.90 , means that $90 \%$ of variance in GCV was explained by variance in NIR spectra and $10 \%$ cannot be counted by NIR spectra (unexplained variance). Bias mentions the overall accuracy of the GCV model [26]. The low value of RPD means the measured value is not robust. If the model had a high RMSECV, then that modelling should need an increasing number of the sample set or sometimes it is seen that not necessary to develop model [26].
Fig. 3a and b illustrated comparison of the GCV of maize cob milled sample predicted by near infrared spectroscopy and measured by the bomb calorimeter of the calibration model and the validation model. The regression coefficient of GCV was shown in Fig. 4. Shown in Fig. 5 was first derivative spectra of maize cob milled sample used for model development. The obvious peaks occurring on regression coefficient plot and second derivative spectra were at $5801 \mathrm{~cm}^{-1}(1723 \mathrm{~nm})$, $4412 \mathrm{~cm}^{-1}(2266 \mathrm{~nm})$, and $4293 \mathrm{~cm}^{-1}(2329 \mathrm{~nm})$, related to $\mathrm{C}-\mathrm{H}$ methylene $\left(\mathrm{CH}_{2}\right)$ of hydrocarbons, O-H stretching/C-O stretching combination of cellulose, and $\mathrm{C}-\mathrm{H}$ stretching $/ \mathrm{CH}_{2}$ deformation combination of starch [6].

As reported by Posom and Sirisomboon [9], the band of cellulose and fiber had high impact to the forecast of the $\mathrm{HV}$ of the oil extracted residue of Jatropha curcas kernels. In addition, Zhang et al. [10] also reported that the important band for estimating $\mathrm{HHV}$ of sorghum was the $\mathrm{C}-\mathrm{H}$ stretching and $\mathrm{CH}_{3}$ structure.

To confirm whether calibration model can estimate the GCV of future sample, the 10 external samples were tested using calibration model, from which the outcome was shown in Table 2. The performance test had a low RMSEP and bias with $77.3 \mathrm{Jg}^{-1}$ and $-13.7 \mathrm{Jg}^{-1}$, respectively.

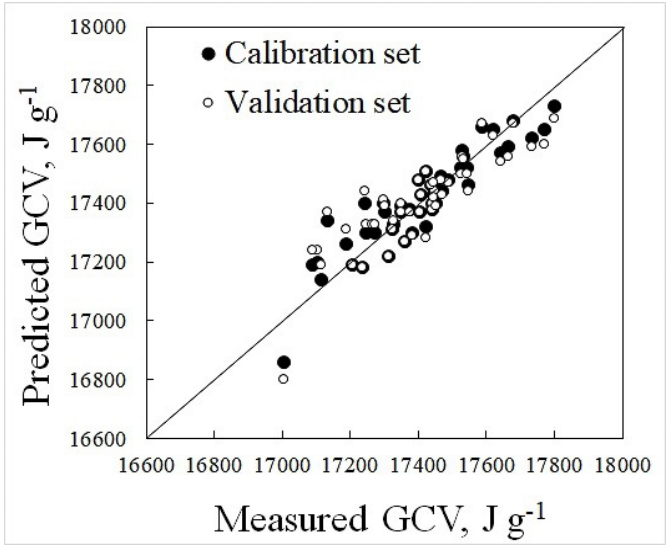

Fig. 3. Comparison of the gross calorific of maize cob milled sample predicted by near infrared spectroscopy and measured by the reference laboratory of the calibration model and the validation model.

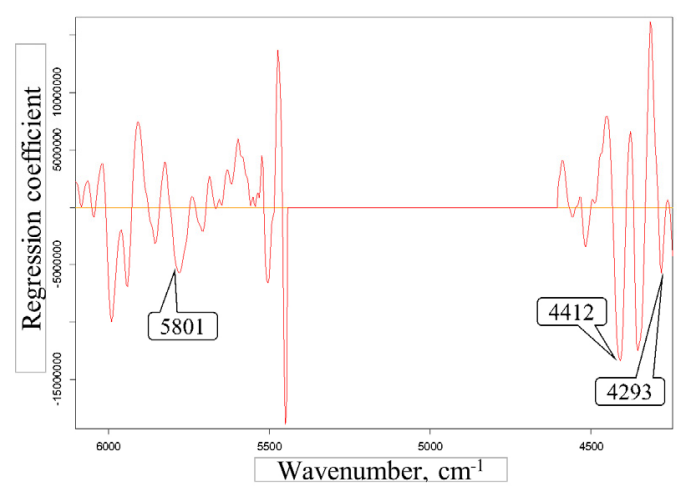

Fig. 4. PLS model regression coefficient plot for the gross calorific of maize cob milled sample. 


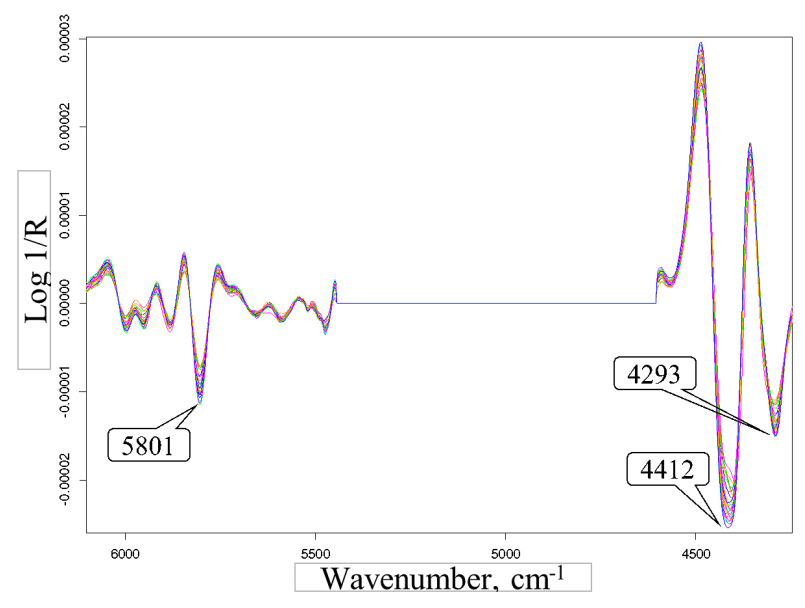

Fig. 5. First derivative spectra of maize cob milled sample used for model development.

Table 2. Measured value and predicted value of external sample predicted by calibration model

\begin{tabular}{|c|c|c|}
\hline Sample & TRUE $\left(\mathbf{J g}^{-1}\right)$ & Prediction $\left.\mathbf{( J ^ { - 1 }}{ }^{-1}\right)$ \\
\hline 51 & 17431.0 & 17375 \\
\hline 52 & 17305.5 & 17430 \\
\hline 53 & 17404.0 & 17387 \\
\hline 54 & 17327.5 & 17242 \\
\hline 55 & 17387.5 & 17388 \\
\hline 56 & 17348.5 & 17403 \\
\hline 57 & 17368.0 & 17438 \\
\hline 58 & 17422.0 & 17522 \\
\hline 59 & 17253.0 & 17141 \\
\hline 60 & 17264.5 & 17322 \\
\hline RMSEP $\left(\mathrm{Jg}^{-1}\right)$ & \multicolumn{2}{|c|}{77.3} \\
\hline Bias $\left(\mathrm{Jg}^{-1}\right)$ & \multicolumn{2}{|c}{} \\
\hline
\end{tabular}

\section{Conclusions}

The outcome showed that there is quite a possibility to the use of NIR spectroscopy as a rapid technique for classifying maize cob properties with a fair performance. Moreover, the time per sample can be reduced to 2-3 minutes where the current analysis took approximately 25-30 minutes per sample. For robustness, the model was tested by external sample (unknown sample) to confirm its ability again because it is the representative population for future. For model development, the vibrational band of hydrocarbons, cellulose, and starch strongly had an impact to the prediction of GCV. In the further study for improvement of the calibration model performance, the mode development should be done by collecting maize cob from various sources to get wider range of $\mathrm{GCV}$, that way the calibration model may be a robust and global model.

\section{Acknowledgments}

The authors thank the Near Infrared Spectroscopy Research Center for Agricultural Product and Food at
King Mongkut's Institute of Technology Ladkrabang, Bangkok, Thailand, for the use of their instruments. We also acknowledge the financial support from the King Mongkut's Institute of Technology Ladkrabang research fund.

\section{References}

1. Posom, J., and Sirisomboon, P. 2017. Evaluation of lower heating value and elemental composition of bamboo using near infrared spectroscopy. Energy $121,147-158$.

2. Posom, J., Shrestha, A., Saechua, W. and Sirisomboon, P. 2016. Rapid non-destructive evaluation of moisture content and higher heating value of Leucaena leucocephala pellets using near infrared spectroscopy. Energy 107, $464-472$.

3. Everard, C. D., McDonnell, K. P. and Fagan, C. C. 2012. Prediction of biomass gross calorific values using visible and near infrared spectroscopy. Biomass and Bioenergy (45), $203-211$.

4. Huang, C., Han, L., Yang, Z. and Liu, X. 2009. Ultimate analysis and heating value prediction of straw by near infrared spectroscopy. Waste Management (29), 1793 - 1797.

5. J. Workman, J.R.L. Weyer. 2007 Practical Guide to Interpretive Near-infrared Spectroscopy, Taylor and Francis, Boca Raton, FL, pp. 240-262.

6. John S. Shenk, Jerome J. Workman, Jr., and Mark O. Westerhaus. 2008. Application of NIR Spectroscopy to Agricultural Products. Text book: Editor: Donald A. Burns Emil W. Ciurczak. Hand book of NearInfrared Analysis. CRC press. Taylor \& Francis Group. pp. 356-357.

7. R. Zornoza, C. Guerrero, J. Mataix-Solera, KM. Scow, V. Arcenegui, J.M. Beneyto. 2008. Near infrared spectroscopy for determination of various physical, chemical and biochemical properties in Mediterranean soils, Soil Biol Biochem. 40 (7). 301923.

8. P. Williams. 2007. Near-Infrared Technology Getting the Best Out of Light Edition 5.0. A Short Course in the Practical Implementation of Near-infrared Spectroscopy for the User, PDK Grain, Nanaimo, Canada.

9. J. Posom, P. Sirisomboon. 2015. Evaluation of the moisture content of Jatropha curcas kernels and the heating value of the oil-extracted residue using nearinfrared spectroscopy, Biosyst Eng. 130. 52-59.

10. K. Zhang, L. Zhou, M. Brady, F. Xu, J. Yu, D. Wang. 2017. Fast analysis of high heating value and elemental compositions of sorghum biomass using near-infrared spectroscopy, Energy. 118. 1353-1360. 